DOI 10.4171/JEMS/200

Marston D. E. Conder · Jozef Širáň · Thomas W. Tucker

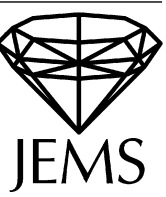

\title{
The genera, reflexibility and simplicity of regular maps
}

Received November 23, 2007 and in revised form July 9, 2008

\begin{abstract}
This paper uses combinatorial group theory to help answer some long-standing questions about the genera of orientable surfaces that carry particular kinds of regular maps. By classifying all orientably-regular maps whose automorphism group has order coprime to $g-1$, where $g$ is the genus, all orientably-regular maps of genus $p+1$ for $p$ prime are determined. As a consequence, it is shown that orientable surfaces of infinitely many genera carry no regular map that is chiral (irreflexible), and that orientable surfaces of infinitely many genera carry no reflexible regular map with simple underlying graph. Another consequence is a simpler proof of the Breda-Nedela-Širán classification of non-orientable regular maps of Euler characteristic $-p$ where $p$ is prime.
\end{abstract}

Keywords. Regular map, symmetric graph, embedding, genus, chiral, reflexible

\section{Introduction}

Regular maps are generalizations of the platonic solids (viewed as tessellations of the sphere) to surfaces of higher genus. Their formal study was initiated by Brahana [4] in the 1920s and continued by Coxeter (see [10]) and others decades later. Regular maps on the sphere and the torus and other orientable surfaces of small genus are now quite well understood, but until recently, the situation for surfaces of higher genus has been something of a mystery. In particular, some long-standing questions have remained open, about the genera of orientable surfaces carrying a regular map having no multiple edges, or a regular map that is chiral (admitting no reflectional symmetry). This paper takes a significant step towards answering these questions.

Here, a map $M$ is an embedding of a connected graph or multigraph into a closed surface, such that each component (or face) of the complement is simply connected. The genus and the Euler characteristic of the map $M$ are defined as the genus and the Euler

M. D. E. Conder: Department of Mathematics, University of Auckland, Private Bag 92019, Auckland, New Zealand; e-mail: m.conder@auckland.ac.nz

J. Širáň: Department of Mathematics, SvF, Slovak University of Technology, Bratislava, Slovakia and Open University, Milton Keynes, MK7 6AA, UK; e-mail: siran@math.sk

T. W. Tucker: Mathematics Department, Colgate University, Hamilton, NY 13346, U.S.A.; e-mail: ttucker@mail.colgate.edu

Mathematics Subject Classification (2010): 05C25, 57M15 
characteristic of the supporting surface. The topological dual of $M$ (which is denoted by $M^{*}$ ) is obtained from $M$ by interchanging the roles of vertices and faces in the usual way.

An automorphism of a map $M$ is any permutation of the edges of the underlying (multi)graph that preserves the embedding, or equivalently, any automorphism of the (multi)graph induced by a homeomorphism of the supporting surface. By connectedness, any automorphism is uniquely determined by its effect on any flag (which is an incident vertex-edge pair $(v, e)$ taken together with a chosen side along the edge $e$ ). The automorphism group of $M$ is denoted by $\operatorname{Aut}(M)$. If the surface is orientable, then the subgroup of $\operatorname{Aut}(M)$ of all orientation-preserving automorphisms is denoted $\operatorname{Aut}^{o}(M)$, and this has index at most 2 in $\operatorname{Aut}(M)$; if $M$ admits an orientation-reversing automorphism (so that $\operatorname{Aut}^{o}(M)$ has index 2 in $\operatorname{Aut}(M)$ ), then $M$ is said to be reflexible, and otherwise $M$ is chiral. If the surface is non-orientable, then there is no such distinction.

An orientable map $M$ is called regular (or orientably-regular, or sometimes rotary) if $G=\operatorname{Aut}^{o}(M)$ acts regularly on the set of oriented edges (or arcs) of $M$. The platonic solids give the most famous examples. If each face has size $k$ and each vertex has valence $m$, then the map $M$ is said to have type $\{k, m\}$, and $M$ is regular if and only if there is a $k$-fold rotation $X$ about the centre of a face $f$ and an $m$-fold rotation $Y$ about an incident vertex $v$, with product $X Y$ an involutory rotation around the midpoint of an edge $e$ incident with $v$ and $f$. By connectedness, $X$ and $Y$ generate $G=\operatorname{Aut}^{\circ}(M)$, which is therefore a quotient of the ordinary $(k, m, 2)$ triangle group $\Delta^{o}(k, m, 2)=\left\langle x, y, z \mid x^{k}=y^{m}=z^{2}=x y z=1\right\rangle$ (under an epimorphism taking $x$ to $X$ and $y$ to $Y$ ). The dual $M^{*}$ is also regular, with the roles of $X$ and $Y$ interchanged, and the map $M$ (or its dual $M^{*}$ ) is reflexible if and only if the group $G=\operatorname{Aut}^{o}(M)$ admits an automorphism of order 2 taking $X$ to $X^{-1}$ and $Y$ to $Y^{-1}$, or equivalently (following conjugation by $X$ ), an automorphism of order 2 taking $X$ to $X^{-1}$ and $X Y$ to $Y^{-1} X^{-1}=(X Y)^{-1}=X Y$.

Conversely, given any epimorphism $\theta$ from $\Delta^{o}(k, m, 2)$ to a finite group $G$ with torsion-free kernel, a map $M$ can be constructed using (right) cosets of the images of $\langle x\rangle,\langle y\rangle$ and $\langle z\rangle$ as vertices, faces and edges, with incidence given by non-empty intersection, and then $G$ acts regularly on the ordered edges of $M$ by (right) multiplication. From this point of view the study of regular maps is simply the study of smooth quotients of triangle groups-with 'smooth' here meaning that the orders of the generators $x, y$ and $z$ are preserved.

Deep connections exist between maps and other branches of mathematics, however, which go far beyond group theory, and include hyperbolic geometry, Riemann surfaces and, rather surprisingly, number fields and Galois theory. A brief summary can be given as follows. If $1 / k+1 / m<1 / 2$, then the $(k, m, 2)$-triangle group acts as a group of hyperbolic isometries preserving an infinite tessellation of the hyperbolic plane consisting of congruent $k$-gons, with $m$ of them meeting at each vertex. Factoring out by any torsion-free normal subgroup of finite index yields (as quotient space) a regular map of type $\{k, m\}$ on some compact, orientable surface, endowed with hyperbolic geometry and complex structure from the Poincaré metric on the complex upper half-plane (on which 
the triangle group acts). If the normality condition is dropped, one still obtains maps (not necessarily regular) on compact surfaces. Maps can thus be viewed as complex algebraic curves; moreover, by substantial results in algebraic varieties, the curves can be taken to be defined over algebraic number fields.

This connection has opened up the possibility of studying the absolute Galois group by its action on maps defined in terms of the natural action of the group on coefficients of the defining polynomial of the corresponding Riemann surface over an algebraic number field, as suggested in Grothendieck's programme [11]. Further details about these and other exciting connections can be found in excellent survey papers by Jones [14] and Jones and Singerman [15].

By a celebrated theorem of Hurwitz, for any $g \geq 2$ the order of a finite group acting as a group of conformal automorphisms of the Riemann surface of genus $g$ is bounded above by $84(g-1)$. A classical problem here is classification of the largest possible group of automorphisms for any given genus $g \geq 2$. As was shown by Accola [1], this problem reduces to a large extent, for infinitely many genera, to the classification of all regular maps on a surface of given genus. For example, the case where $g=p+1$ for some prime $p$ and the group has order greater than $6(g-1)$ has been dealt with by Belolipetsky and Jones [2], and involves three families of chiral maps (which will reappear in Section 3 of this paper).

It is well known that for every $g>0$ there exists a reflexible regular map of type $\{4 g, 4 g\}$ on an orientable surface of genus $g$ (with dihedral automorphism group). It follows that there are no 'gaps' in the genus spectrum of orientable surfaces carrying reflexible regular maps. On the other hand, the underlying graphs for these maps are highly degenerate, being bouquets of $2 g$ loops based at a single vertex.

There is no analogous family for non-orientable surfaces. Indeed, it has been known for some time that there are gaps in the the genus spectrum of non-orientable regular maps, at least for small genera (see [18], for example). This has led to a fundamental question about whether or not there exist infinitely many gaps in the genus spectrum of non-orientable regular maps. A similar question has been raised about the genus spectrum of orientably-regular but chiral maps.

On the positive side, the connection between regular maps and smooth quotients of triangle groups has been exploited on occasion to construct various infinite families of regular maps, with the aim of filling possible gaps. For example, this approach was taken by Conder and Everitt [8] to prove that non-orientable surfaces of more than 75 per cent of all genera carry some regular map. The same kind of approach, sometimes with the help of group-theoretic procedures now available in systems like MAGMA [3], has been taken successfully to determine all regular maps of various small genera, including all orientably-regular maps of genus 2 to 15; see [7] and references therein.

Until very recently, however, there has been no significant classification of regular maps by supporting surface for an infinite family of genera. A major breakthrough was achieved in a classification by Breda d'Azevedo, Nedela and Širán [5] of regular maps on non-orientable surfaces of Euler characteristic $-p$, for $p$ an odd prime. One consequence of this classification is that there are no such maps for $p \equiv 1 \bmod 12$, thereby exhibiting infinitely many gaps in the genus spectrum of non-orientable regular maps. This has been 
taken further by Conder, Potočnik and Širáň [9], in showing that there are no regular maps of Euler characteristic $-p^{2}$ for any prime $p>7$.

Recently, Conder extended the computer-assisted search for all regular maps of small Euler characteristic, using a new version of an algorithm for finding all normal subgroups of up to a given index in a finitely-presented group; see [6]. The resulting lists of all regular maps of characteristic -1 to -200 reveal patterns in the genus spectrum of various kinds of regular maps never seen before, and these have led us to the main observations that we prove in this paper.

As in [5], if one attempts to classify regular maps on some orientable surface of genus $g$, one may begin with the Euler-Poincaré formula

$$
2-2 g=\chi=V-E+F=|G|\left(\frac{1}{k}-\frac{1}{2}+\frac{1}{m}\right),
$$

which relates the type $\{k, m\}$ of the map $M$ and the order of $G=\operatorname{Aut}^{o}(M)$ to the genus $g$. This yields an equation in integers with $|G|$ and $g-1$ appearing on different sides of the equation. Two natural extremes arise that one has to consider: the case where $g-1$ divides $G$ on one hand, and the case where $g-1$ and $|G|$ are relatively prime on the other.

In this paper we apply combinatorial group theory (sometimes in a remarkably elementary way) to prove a number of results in this direction. First, we classify all orientably-regular maps $M$ of genus $g>1$ such that $g-1$ is a prime dividing $\left|\operatorname{Aut}^{o}(M)\right|$. Second, we make a major advance by producing a classification of all orientably-regular maps $M$ of genus $g \geq 0$ for which $g-1$ and $\mid$ Aut $^{o}(M) \mid$ are relatively prime. As a consequence of these results, we obtain a complete classification of all orientably-regular maps $M$ of genus $p+1$ where $p$ is prime, and as corollaries, we have the following:

(1) If $p$ is a prime such that $p-1$ is not divisible by 3,5 or 8 , then every orientablyregular map of genus $g=p+1$ is reflexible.

(2) If $M$ is an orientably-regular but chiral map of genus $g=p+1$, where $p$ is prime, and $p-1$ is not divisible by 5 or 8 , then either $M$ or its topological dual $M^{*}$ has multiple edges.

(3) If $M$ is a reflexible orientably-regular map of genus $g=p+1$, where $p$ is prime and $p>13$, then either $M$ or $M^{*}$ has multiple edges, and if $p \equiv 1 \bmod 6$, then both $M$ and $M^{*}$ have multiple edges.

The first of these shows there exist infinitely many gaps in the spectrum of orientablyregular maps that are chiral, and the third shows there exist infinitely many gaps in the spectrum of reflexible orientably-regular maps that have simple underlying graphs. We also obtain from this work a new and more concise proof of the classification result of [5] for non-orientable maps.

The fact that we could take a common approach to considering genus questions for simplicity, reflexibility, and non-orientability, we found surprising. We acknowledge the use of MAGMA in helping us obtain these results, and for showing us the best way forward in some of the proofs. 


\section{Preliminaries}

For an orientably-regular map $M$ of type $\{k, m\}$, we will always let $G=\operatorname{Aut}^{o}(M)$, which is generated by single-step rotations $X$ and $Y$ about a face and incident vertex, such that $X Y$ reverses an incident edge. Thus $X^{k}=Y^{m}=(X Y)^{2}=1$.

We will define the $X$-core of $G$ to be the largest normal subgroup of $G$ contained in $\langle X\rangle$ and denote this by $\operatorname{Co}(X)$, and define the $Y$-core $\operatorname{Co}(Y)$ similarly. Note that if $\langle Y\rangle$ stabilizes the vertex $v$, then the stabilizers of the neighbours of $v$ are conjugates of $\langle Y\rangle$ by $X Y^{i}$ for $0 \leq i<m$, so the existence of multiple edges incident to $v$ is equivalent to having $\langle Y\rangle^{X Y^{i}}=\langle Y\rangle^{X Y^{j}}$ for some $i \neq j$, which in turn is easily seen to be equivalent to $\operatorname{Co}(Y)$ being non-trivial. We will say the group $G$ is non-degenerate if both $\operatorname{Co}(X)$ and $\operatorname{Co}(Y)$ are trivial, degenerate if $\operatorname{Co}(X)$ or $\operatorname{Co}(Y)$ is non-trivial, singly-degenerate if exactly one of them is non-trivial, and doubly-degenerate if both are non-trivial. If $G$ is non-degenerate (resp. singly-degenerate, or doubly-degenerate) then both (resp. exactly one of, or neither of) the map $M$ and its dual $M^{*}$ will have simple underlying graph.

Further, we will say that $G$ is $X Y$-disjoint if $\langle X\rangle \cap\langle Y\rangle=\{1\}$. Note that if $G$ is non-degenerate, then it must be $X Y$-disjoint, but not conversely.

We will make extensive use of a number of key theorems from group theory:

Theorem 2.1 (Schur-Zassenhaus theorem). If $N$ is a normal subgroup of the finite group $G$, such that the order $|N|$ and the index $|G: N|$ are coprime, then $G$ contains a subgroup of order $|G: N|$, and any two such subgroups are conjugate in $G$.

Theorem 2.2 (Transfer to a central subgroup). If $H$ is a subgroup of the centre $Z(G)$ of the group $G$, with finite index $|G: H|=m$, then the mapping $\tau: G \rightarrow G$ given by $x \mapsto x^{m}$ (for all $x \in G$ ) is a homomorphism.

Corollary 2.3 (Schur's theorem). If the centre $Z(G)$ of a group $G$ has finite index $m$, then the commutator subgroup $G^{\prime}$ is finite and the order of every element of $G^{\prime}$ divides $m$.

Theorem 2.4 (Ito's theorem). If the group $G$ is expressible as $A B$ where $A$ and $B$ are abelian subgroups of $G$, then the commutator subgroup $G^{\prime}$ is abelian (and hence $G$ is solvable).

The proofs of the first three of these can be found in [16. Chapters $9 \& 10$ ], and the fourth in [12].

We will also require the classification of non-solvable, almost Sylow-cyclic groups. A finite group $G$ is said to be almost Sylow-cyclic if all its Sylow subgroups of odd order are cyclic and (when $|G|$ is even) all its Sylow 2-subgroups have a cyclic subgroup of index 2. Classification of all solvable almost Sylow-cyclic groups is a classical result by Zassenhaus. The non-solvable case is covered by the results of Suzuki [17] and Wong [19], giving the following:

Theorem 2.5 (Suzuki and Wong). Let $G$ be a finite, non-solvable, almost Sylow-cyclic group. Then $G$ has a subgroup $G_{0}$ of index at most 2 such that $G_{0}$ is isomorphic to $H \times L$, where $H$ is isomorphic to a semi-direct product of two cyclic groups of order $u$ and $v$, and $L \cong \operatorname{SL}(2, q)$ or $\operatorname{PSL}(2, q)$ for some prime $q>3$, with $u$, $v$ and $|L|$ being pairwise relatively prime. 
Finally, we need the following important observations, the second using the fact that if $G$ is abelian then $X=\operatorname{Co}(X)$ and $Y=\operatorname{Co}(Y)$ so $G$ is doubly-degenerate. Here, and later, $C_{G}(H)$ denotes the centralizer of the subgroup $H$ in the group $G$.

Lemma 2.6. Every element of $\operatorname{Co}(X)$ and every element of $\operatorname{Co}(Y)$ commutes with all squares in $G$. In particular, if $Y$ has odd order, then $\operatorname{Co}(X)$ is central in $G$.

Proof. Consider the homomorphism $\theta: G \rightarrow \operatorname{Aut}(\operatorname{Co}(Y))$ given by conjugation. As $G=\langle X Y, Y\rangle$, the $\theta$-image of $X Y$ generates $\operatorname{im} \theta$, so this has order at most 2 . Hence all squares and all elements of odd order in $G$ lie in $\operatorname{ker} \theta=C_{G}(\operatorname{Co}(Y))$. The same is true for $\operatorname{Co}(X)$. In particular, if $Y$ has odd order then $G=\langle X, Y\rangle \subseteq C_{G}(\operatorname{Co}(X))$.

Lemma 2.7. If $G$ has a normal cyclic subgroup $N$, then either $G$ is degenerate or $2|N|$ divides the Euler characteristic $\chi$ of the map $M$.

Proof. Suppose $G$ is non-degenerate. Then $X Y \notin N$, for otherwise $X Y$ would be the only involution in the cyclic normal subgroup $N$, making $X Y$ central, and then $G=\langle X, X Y\rangle$ would be abelian and hence degenerate. Also $\langle X\rangle \cap N$ and $\langle Y\rangle \cap N$ are characteristic in the cyclic subgroup $N$ and so normal in $G$, hence both are trivial. Thus $G / N$ is the orientation-preserving group of automorphisms of an orientably-regular map of the same type as $M$. It follows that in applying the Euler-Poincaré formula, we simply replace $|G|$ by $|G / N|$, so this quotient map has Euler characteristic $\chi /|N|$, which is an even integer, and hence $2|N|$ divides $\chi$.

\section{The case where $p$ divides $|G|$}

In this section we find all possibilities for an orientably-regular map $M$ of genus $p+1$ where $p$ is prime, $p>13$ and $\left|\operatorname{Aut}^{o}(M)\right|$ is divisible by $p$. All such maps turn out to be chiral, and fall into three families (as arose in a slightly different context in [2]).

Theorem 3.1. Let $M$ be an orientably-regular map of genus $g=p+1$ for some prime $p>13$, and let $G=\operatorname{Aut}^{o}(M)$ be its group of orientation-preserving automorphisms. If $p$ divides the order of $G$, then one of the following holds:

(a) $M$ has type $\{8,8\}$ and $|G|=8 p$, with $p \equiv 1 \bmod 8$,

(b) $M$ has type $\{5,10\}$ and $|G|=10 p$, with $p \equiv 1 \bmod 10$,

(c) $M$ has type $\{6,6\}$ and $|G|=12 p$, with $p \equiv 1 \bmod 6$.

Moreover, in each of these three cases $M$ is chiral, and in cases (a) and (b) the group $G$ is non-degenerate, while in case (c) it is singly-degenerate. Up to equivalence and duality there is one chiral pair of such maps for each $p$ in cases (a) and (c), and there are two chiral pairs for each $p$ in case (b).

Proof. First suppose $p>84$, and let the map $M$ have type $\{k, m\}$. By the Euler-Poincaré formula, we know that $|G|=R(g-1)=R p$ where $R=4 \mathrm{~km} /(\mathrm{km}-2 \mathrm{k}-2 \mathrm{~m})$ satisfies $4<R \leq 84$. Since $p$ divides $|G|$, we see that $G$ has a unique Sylow $p$-subgroup $P$, 
which is cyclic, and normal in $G$ with index $R$. In particular, since $R$ is the order of $G / P$, we see that $R$ is an even integer, so $R \geq 6$, but $R$ is not divisible by $p$.

Now if $p$ divides $k$ or $m$, then since $p$ does not divide $R=4 \mathrm{~km} /(\mathrm{km}-2 \mathrm{k}-2 \mathrm{~m})$, we find that $p$ has to divide both $k$ and $m$, and hence $p^{2}$ divides $k m-2 k-2 m$, so $p^{2}$ divides $k+m$. On the other hand, since $k$ and $m$ are divisible by $p$, which is greater than 6 , the ratio $R=4 \mathrm{~km} /(\mathrm{km}-2 \mathrm{k}-2 \mathrm{~m})$ is less than 12 , and hence $|G|=R p<12 p$. In particular, each of $k$ and $m$ is less than $12 p$, so $k+m<24 p$, which makes it impossible for $p^{2}$ to divide $k+m$. Thus $p$ divides neither $k$ nor $m$.

Next, let $X$ and $Y$ be generators for $M$ satisfying the usual relations $X^{k}=Y^{m}=$ $(X Y)^{2}=1$. Then each of $\langle X\rangle$ and $\langle Y\rangle$ has trivial intersection with $P$ (since $p$ divides neither $k$ nor $m$ ), as does $\langle X Y\rangle$ (since $|P|=p$ is odd). Hence the quotient $G / P$ is the orientation-preserving group of automorphisms of an orientably-regular map of the same type $\{k, m\}$ as $M$, with Euler characteristic $-2 p / p=-2$, so genus 2 .

The orientably-regular maps of genus 2 are well known, and listed in [7]. In particular, we find from this list that one of the following holds:

(a) $\{k, m\}=\{8,8\}$ and $G / P \cong C_{8}$;

(b) $\{k, m\}=\{5,10\}$ and $G / P \cong C_{10}$;

(c) $\{k, m\}=\{6,6\}$ and $G / P \cong C_{6} \times C_{2}$;

(d) $\{k, m\}=\{4,8\}$ and $G / P \cong C_{8} \rtimes C_{2}$, metacyclic of order 16;

(e) $\{k, m\}=\{4,6\}$ and $G / P \cong C_{3} \rtimes D_{4}$, of order 24 (with centre of order 2);

(f) $\{k, m\}=\{3,8\}$ and $G / P \cong \mathrm{GL}(2,3)$, of order 48 .

We will consider each of these cases in turn, using the following observations.

Since $\operatorname{Aut}(P) \cong \operatorname{Aut}\left(C_{p}\right)$ is cyclic, we know that $G / C_{G}(P)$ is abelian and hence $C_{G}(P)$ contains $G^{\prime}$. On the other hand, $G$ itself is not abelian, for otherwise the order of $G$ would divide $2 k$ and then be coprime to $p$, contradiction.

Now consider case (a). Here $G / P$ is cyclic of order 8 , so $G$ is a semi-direct product of $C_{p}$ by $C_{8}$. As $Y$ has order 8 and $X Y$ has order 2 (but $G$ itself is not cyclic) we have $X Y=w Y^{4}$ for some non-trivial element $w$ of $P$, and indeed $w$ must generate $P$ (since $|P|$ is prime). Also $1=(X Y)^{2}=w\left(w^{Y^{4}}\right)$, so $Y^{4}$ conjugates $w$ to $w^{-1}$, and hence conjugation by $Y$ induces an automorphism of $P$ of order 8. In particular, $p$ is congruent to $1 \bmod 8$. Also $X=X Y Y^{-1}=w Y^{3}$. Note there are four possibilities: if $c$ is a primitive 8th root of 1 in $\mathbb{Z}_{p}$, then $Y^{-1} w Y=w^{c^{i}}$ where $i=1,3,5$ or 7 . None of these gives a reflexible map, for if $\tau$ were an automorphism that inverts both $X$ and $Y$, then $\tau$ would take $w=X Y^{-3}$ to $X^{-1} Y^{3}=Y^{-3} w^{-1} Y^{3}=\left(w^{-1}\right)^{Y^{3}}$, and then could not have order 2 . Hence these four possibilities fall into two chiral pairs, with one pair dual to the other. Also in each case it is easy to see that $\langle X\rangle$ and $\langle Y\rangle$ are both core-free, so the map $M$ and its dual both have simple underlying graphs. Hence in case (a), up to equivalence and duality we have just one chiral pair of non-degenerate maps of type $\{8,8\}$ and genus $p+1$, whenever $p \equiv 1 \bmod 8$.

Similarly, in case (b) we know $G / P$ is cyclic (of order 10), so $G$ is a semi-direct product of $C_{p}$ by $C_{10}$, and assuming $X$ has order 5 while $Y$ has order 10, we have $X Y=$ $w Y^{5}$ for some generator $w$ of $P$. Also $1=(X Y)^{2}=w\left(w^{Y^{5}}\right)$, so conjugation by $Y^{5}$ inverts $w$. Furthermore, $X=X Y Y^{-1}=w Y^{4}$, and as $X$ has order 5, we see that $Y^{4}$ cannot 
centralize $w$ (or else $X=w Y^{4}$ would have order $5 p$ ), and hence conjugation by $Y$ induces an automorphism of $P$ of order 10. In particular, $p$ is congruent to $1 \bmod 10$. Again there are four possibilities: if $c$ is a primitive 10th root of 1 in $\mathbb{Z}_{p}$, then $Y^{-1} w Y=w^{c^{i}}$ where $i=1,3,7$ or 9 . All give irreflexible maps, by the same kind of argument as in case (a), and again both $\langle X\rangle$ and $\langle Y\rangle$ are core-free. Hence in case (b), up to equivalence we have two chiral pairs of non-degenerate maps of type $\{5,10\}$ and genus $p+1$, whenever $p \equiv 1$ $\bmod 10$.

In case (c), the quotient $G / P$ is isomorphic to $C_{6} \times C_{2}$. Now $P$ cannot be centralized by $X Y$ (of order 2), for otherwise $\langle X Y, P\rangle$ would be an abelian normal subgroup of order $2 p$ in $G$, with unique involution $X Y$, and then $X Y$ would be central in $G$, making $G=\langle X Y, X\rangle$ abelian. Similarly, $P$ cannot be centralized by $X^{2}$ or $Y^{2}$ (of order 3), for otherwise $C_{G}(P)$ would contain a characteristic subgroup $K$ of order 3 that would then be normal in $G$, and the quotient $G / K$ of order $4 p$ would be $(2,2,2)$-generated, which is impossible. On the other hand, $G / C_{G}(P)$ is isomorphic to a subgroup of $\operatorname{Aut}(P)$ and is therefore cyclic, and is a quotient of $G / P \cong C_{6} \times C_{2}$, so must be cyclic of order 6 . It follows that $p \equiv 1 \bmod 6$, and that conjugation by $X Y$ inverts every element of $P$, and conjugation by either $X$ or $Y$ induces an automorphism of $P$ of order 6 . Without loss of generality (by moving to the dual if necessary), we may suppose that conjugation of $P$ by $Y$ has order 6, with $Y^{3}$ inverting every element of $P$. Then $X Y^{4}=(X Y) Y^{3}$ centralizes $P$, so conjugation by $X$ induces the same automorphism of $P$ as conjugation by $Y^{-4}=Y^{2}$, and therefore $X^{3}$ centralizes $P$. Thus $C_{G}(P)=\left\langle X^{3}, P\right\rangle$, of order $2 p$. In particular, $\left\langle X^{3}\right\rangle$ is normal in $G$, so $\langle X\rangle$ is not core-free. On the other hand, clearly $\langle Y\rangle$ is core-free. As before, however, both the map and its dual are irreflexible, since if $\tau$ were an automorphism of $G$ inverting both $X$ and $Y$, then $\tau$ would take $w=X^{2} Y^{-4}$ to $w=X^{-2} Y^{4}=Y^{-4} w^{-1} Y^{4}$, and so $\tau$ could not have order 2. In fact there are two possibilities: if $c$ is a primitive 6th root of 1 in $\mathbb{Z}_{p}$, and $w$ is a generator of $P$, then $Y w Y^{-1}=w^{c^{ \pm 1}}$. Hence up to equivalence and duality we have just one chiral pair of singly-degenerate maps of type $\{6,6\}$ and genus $p+1$, whenever $p \equiv 1$ $\bmod 6$.

On the other hand, cases (d) to (f) are impossible. To see this, first note that $|P|$ and $|G: P|$ are coprime, so by the Schur-Zassenhaus theorem, $C_{G}(P)$ has a subgroup $K$ of order $\left|C_{G}(P): P\right|$, and hence $C_{G}(P)$ is a direct product $K \times P$. Each of $K$ and $P$ is characteristic in $C_{G}(P)$ and so is normal in $G$, and $G / K$ is a quotient of $G$ of order $\left|G: C_{G}(P)\right||P|$. In case (d), the abelianization of $G / P$ can be seen from [7, map R2.3] to be isomorphic to $C_{2} \times C_{2}$, and it follows that the largest cyclic quotient of $G$ has order 2 . Thus $C_{G}(P)$ has index 1 or 2 in $G$, so $G / K$ has order $p$ or $2 p$, which is impossible for a quotient of a $(4,8,2)$-generated group. Cases (e) and (f) are even easier: $G / C_{G}(P)$ is a cyclic quotient of the ordinary $(4,6,2)$ or $(3,8,2)$ triangle group and so has order at most 2, so $\left|G: C_{G}(P)\right|=1$ or 2 , and $G / K$ has order $p$ or $2 p$, again a contradiction.

This completes the proof for $p>84$. For $13<p<84$, we may refer to the lists of all orientably-regular maps of genus 2 to 100 computed by Conder [6], or use the same arguments as in the paper [2] by Belolipetsky and Jones. 


\section{The coprime classification: $X Y$-disjoint case}

In this section we begin to consider the case where $|G|$ is coprime to $-\chi / 2=g-1$, where $g$ is the genus of the respective orientably-regular map $M$. We will suppose that $M$ has at least one edge, so that $|G|$ is even, and hence $g-1$ must be odd. The Euler-Poincaré formula gives

$$
4 k m(g-1)=-2 k m \chi=|G|(k m-2 k-2 m) .
$$

Dividing by $d=\operatorname{gcd}(k, m)$, we have

$$
4 \operatorname{lcm}(k, m)(g-1)=|G|(k m / d-2 k / d-2 m / d) .
$$

Since $\operatorname{gcd}(|G|, g-1)=1$, this gives $4 \operatorname{lcm}(k, m)=|G| t$, where $t$ is a positive integer. On the other hand, since $k$ and $m$ are element orders, $\operatorname{lcm}(k, m)$ divides $|G|$, and hence $t$ divides 4 , so $t=1,2$ or 4 . In fact, $t=(k m / d-2 k / d-2 m / d) /(g-1)$, which will be odd if and only if $\mathrm{km} / \mathrm{d}-2 \mathrm{k} / \mathrm{d}-2 \mathrm{~m} / \mathrm{d}$ is odd, and hence $t=1$ if and only if $k$ and $m$ are both odd. Thus $|G|=4 \operatorname{lcm}(k, m)$ whenever $k$ and $m$ are both odd, while $|G|=\operatorname{lcm}(k, m)$ or $|G|=2 \operatorname{lcm}(k, m)$ if at least one of $k$ and $m$ is even.

Next, we show that the group $G$ is almost Sylow-cyclic. Letting $k=k^{\prime} d$ and $m=$ $m^{\prime} d$, we have $\operatorname{gcd}\left(k, m^{\prime}\right)=\operatorname{gcd}\left(k^{\prime}, m\right)=1$, and

$$
|G|=\frac{4 \mathrm{~cm}(k, m)}{t}=\frac{4 k m}{t d}=\frac{4}{t} k^{\prime} m=\frac{4}{t} k m^{\prime} .
$$

It follows that if $q$ is the largest power of any odd prime divisor of $|G|$, then either $q$ divides $k$ but not $m^{\prime}$, or $q$ divides $m$ but not $k^{\prime}$, and in particular, $\langle X\rangle$ or $\langle Y\rangle$ contains a cyclic Sylow $q$-subgroup of $G$. For Sylow 2-subgroups, the situation is similar: if both $k$ and $m$ are odd, then $t=1$, and a Sylow 2-subgroup of $G$ has order 4, so contains a cyclic subgroup of index 2; otherwise $|G|=k^{\prime} m, \mathrm{~km}^{\prime}, 2 \mathrm{k}^{\prime} \mathrm{m}$ or $2 \mathrm{~km}^{\prime}$, and $\langle X\rangle$ or $\langle Y\rangle$ contains a cyclic subgroup of index 1 or 2 in a Sylow 2-subgroup of $G$.

In the special case where $G$ is $X Y$-disjoint, the elements $X^{i} Y^{j}$ are all distinct for $0 \leq i<k$ and $0 \leq j<m$, so $k m \leq|G| \leq 4 \mathrm{lcm}(k, m)=4 \mathrm{~km} / d$, and therefore $d \leq 4$. Moreover, since $|G|=4 \mathrm{lcm}(k, m)$ only when $k$ and $m$ are odd, in which case $d=\operatorname{gcd}(k, m)$ is odd, it follows that $d \leq 3$. On the other hand, if at least one of $k$ and $m$ is even, then $\mathrm{km} \leq|G| \leq 2 \mathrm{~cm}(k, m)=2 \mathrm{~km} / d$, so $d \leq 2$, and hence if $d=3$ then $k$ and $m$ are both odd. Similarly, if $d=2$, then the inequality $k m \leq|G| \leq 2 \operatorname{lcm}(k, m)$ forces $|G|=\mathrm{km}$, which in turn implies that $|G|=2 \mathrm{~km}$ if and only if $k$ and $m$ have opposite parity.

Summarizing, and without loss of generality (by taking the dual if necessary), we have the following possibilities when the group $G$ is $X Y$-disjoint:

(A) $|G|=k m$, where $\operatorname{gcd}(k, m)=1$ or 2 , and $k$ is even,

(B) $|G|=2 \mathrm{~km}$, where $\operatorname{gcd}(k, m)=1$, and $k$ is even but $m$ is odd,

(C) $|G|=4 \mathrm{~km} / 3$, where $\operatorname{gcd}(k, m)=3$, and $k, m$ are both odd,

(D) $|G|=4 \mathrm{~km}$, where $\operatorname{gcd}(k, m)=1$, and $k, m$ are both odd. 
We will classify the possibilities for all groups of each of these types (not just those corresponding to maps for which $g-1$ and $|G|$ are coprime), and give explicit presentations for the group $G$ in each case. In Section 8 we will drop the assumption that $G$ is $X Y$-disjoint, to deal with the general case.

First, for each of the above types we consider the possibilities for the abelianization $G / G^{\prime}$, where $G^{\prime}$ is the commutator subgroup of $G$.

Lemma 4.1. If $G$ is $X Y$-disjoint, then $G / G^{\prime}$ is isomorphic to a subgroup of $C_{2} \times C_{2}$ for type (A), of $C_{2}$ for type (B), or of $C_{3}$ for type (C), while $G / G^{\prime}$ is trivial (and so $G$ is perfect) for type (D).

Proof. These possibilities follow from the fact that $G / G^{\prime}$ is an abelian group generated by the images of $X$ and $Y$, of orders dividing $k$ and $m$, with product of order dividing 2 . For type (A), we have $G / G^{\prime} \cong 1, C_{2}$ or $C_{2} \times C_{2}$ since $\operatorname{gcd}(k, m)=1$ or 2 ; for type (B), $G / G^{\prime} \cong 1$ or $C_{2} \operatorname{since} \operatorname{gcd}(k, m)=1$; for type $(\mathrm{C}), G / G^{\prime} \cong 1$ or $C_{3} \operatorname{since} \operatorname{gcd}(k, m)=3$ and $k, m$ are both odd; and for type (D), $G / G^{\prime} \cong 1$ since $\operatorname{gcd}(k, m)=1$, and $k, m$ are both odd.

Now for type (A) we have the following:

Lemma 4.2. For coprime positive integers $u$ and $v$, the group with presentation $\langle X, Y|$ $\left.X^{2 u}=Y^{2 v}=(X Y)^{2}=\left[X^{2}, Y^{2}\right]=1\right\rangle$ is a group of order $4 u v$, in which $X Y^{2} X^{-1}=$ $Y^{-2}$ and $Y X^{2} Y^{-1}=X^{-2}$.

Proof. In the given group $G$, let $K$ be the subgroup generated by the elements $X^{2}$ and $Y X^{2} Y^{-1}$ (each of order dividing $u$ ). Note that $Y$ normalizes $K$, with $Y^{2}$ centralizing $K$, since $\left[X^{2}, Y^{2}\right]=1$ and then $Y X^{2} Y^{-1}=Y^{-1} X^{2} Y$. As $(X Y)^{2}=1$ we have $X\left(Y X^{2} Y^{-1}\right) X^{-1}=(X Y) X^{2}(X Y)^{-1}=Y^{-1} X^{-1} X^{2} X Y=Y^{-1} X^{2} Y=Y X^{2} Y^{-1}$, so $X$ centralizes $K$, and therefore $K$ is normal in $G$. Also $K$ is abelian (because $X$ centralizes $Y X^{2} Y^{-1}$ ), and so $|K|$ divides $u^{2}$. Furthermore, the quotient $G / K$ (obtained by adding the relation $X^{2}=1$ ) is dihedral of order $4 v$, so $K$ has index $4 v$ in $G$. Similarly, the subgroup $L$ generated by $Y^{2}$ and $X Y^{2} X^{-1}$ is an abelian normal subgroup of $G$ of index $4 u$ and order dividing $v^{2}$. Since $\operatorname{gcd}(u, v)=1$, it follows that $|G|=4 u v$, with $|K|=v$ and $|L|=u$. Finally, since $G / K$ is dihedral we have $X Y^{2} X^{-1}=w Y^{-2}$ for some $w \in K$, but then $w=X Y^{2} X^{-1} Y^{2}$ lies in $K \cap L$ which is trivial (since $\left.\operatorname{gcd}(u, v)=1\right)$, so $X Y^{2} X^{-1} Y^{2}=1$. Similarly $Y X^{2} Y^{-1} X^{2}=1$.

Theorem 4.3. Suppose that $G$ is $X Y$-disjoint and of type (A). Then either $k=2$ and $G$ is dihedral of order $2 m$ where $m$ is odd, or otherwise $k=2 u$ and $m=2 v$ with $\operatorname{gcd}(u, v)=1$, and $G$ is an extension of $C_{u v}$ by $C_{2} \times C_{2}$, with presentation $\langle X, Y$ । $\left.X^{2 u}=Y^{2 v}=(X Y)^{2}=\left[X^{2}, Y^{2}\right]=1\right\rangle$. In the latter case, $X Y^{2} X^{-1}=Y^{-2}$ and $Y X^{2} Y^{-1}=X^{-2}$, and so $G$ is degenerate in both cases.

Proof. First, since $|G|=k m$, and $G$ is $X Y$-disjoint, we know that $G=\langle X\rangle\langle Y\rangle$, and so by Ito's theorem, $G^{\prime}$ is abelian, and hence $G$ is solvable. Moreover, $G / G^{\prime} \cong C_{2}$ or $C_{2} \times C_{2}$, so $G^{\prime}$ contains both $X^{2}$ and $Y^{2}$, and therefore $\left[X^{2}, Y^{2}\right]=1$. 
Now suppose $G / G^{\prime} \cong C_{2}$. Then without loss of generality we can assume not only that $k$ is even but also that $G^{\prime}$ is generated by $X^{2}$ and $Y$ (with $X Y X=Y^{-1}$ ). In particular, $X^{2}$ commutes with $Y$. Moreover, $m$ is odd and $\operatorname{sog} \operatorname{gcd}(k, m)=1$, for otherwise by Lemma 4.2 the elements $X^{2}$ and $Y^{2}$ would generate a normal subgroup of index 4, with abelian quotient, contradicting the assumption that $G / G^{\prime} \cong C_{2}$. Also $X Y X^{-1}=Y^{-1} X^{-2}=$ $\left(X^{2} Y\right)^{-1}$, and as $X Y X^{-1}$ is conjugate to $Y$ (and so has order $m$ ), while $X^{2} Y$ has order $(k / 2) m$, we find that $k / 2=1$, so $k=2$. Hence $G$ is dihedral, and then $\operatorname{Co}(Y)=\langle Y\rangle$, so $G$ is degenerate.

Suppose instead that $G / G^{\prime} \cong C_{2} \times C_{2}$. Then $k$ and $m$ must both be even, so $k=2 u$ and $m=2 v$ with $\operatorname{gcd}(u, v)=1$, and then Lemma 4.2 gives $X Y^{2} X^{-1}=Y^{-2}$ and $Y X^{2} Y^{-1}=X^{-2}$. In particular, $\operatorname{Co}(X)=\left\langle X^{2}\right\rangle$ and $\operatorname{Co}(Y)=\left\langle Y^{2}\right\rangle$, so $G$ is degenerate. Also $G^{\prime}$ is generated by the commuting elements $X^{2}$ and $Y^{2}$ of coprime orders $u$ and $v$, so $G^{\prime}$ is cyclic of order $u v$.

In the two cases given by Theorem 4.3 , we will say that $G$ is of type (A1) or (A2), respectively. For the other three types, we proceed thus:

Theorem 4.4. If $G$ is non-degenerate, and of type (B), (C) or (D), then:

(B) $G \cong\left\langle X, Y \mid X^{4}=Y^{3}=(X Y)^{2}=1\right\rangle \cong S_{4}$, or

(C) $G \cong\left\langle X, Y \mid X^{3}=Y^{3}=(X Y)^{2}=1\right\rangle \cong A_{4}$, or

(D) $G \cong\left\langle X, Y \mid X^{3}=Y^{5}=(X Y)^{2}=1\right\rangle \cong A_{5}$.

Proof. First, suppose that $G$ is solvable, and let $J$ be any minimal normal subgroup of $G$. Then $J$ is elementary abelian, and further, since $G$ is almost Sylow-cyclic, $J$ is isomorphic to $C_{q}$ for some prime $q$, or to $C_{2} \times C_{2}$. By Lemma 2.7 however, $J$ cannot be cyclic, and therefore $J \cong C_{2} \times C_{2}$. Moreover, since $G$ is perfect for type (D), that means we only need to consider types (B) and (C).

For type (B), the quotient $G / J$ has order $|G| / 4=(k / 2) m$, where $m$ is odd, so $J$ must contain $X^{k / 2}$, and hence $G / J$ is the product of its subgroups $\langle X\rangle J / J$ and $\langle Y\rangle J / J$, of orders $k / 2$ and $m$, which intersect trivially. By the same argument as in the first part of the proof of the last theorem (for case (A)), we find that $G / J$ is dihedral, so $k / 2=2$, giving $k=4$, and the subgroup $\langle Y\rangle J / J$ is normal in $G / J$. On the other hand, no non-trivial element of $\langle Y\rangle$ can centralize $J$, for otherwise that element would generate a characteristic central subgroup of $\langle Y\rangle J$, and then be normal in $G$, contradicting the assumption that $G$ is non-degenerate. As $J$ is isomorphic to $C_{2} \times C_{2}$, we know $\operatorname{Aut}(J)$ is isomorphic to $S_{3}$, and then since $Y$ has odd order, it follows that $\langle Y\rangle$ has order 3 . Thus $\{k, m\}=\{4,3\}$, and $G \cong S_{4}$, as claimed.

For type (C), we see that $G / J$ has order $|G| / 4=\mathrm{km} / 3$, which is odd, so $X Y \in J$, and hence $G / J=\langle X Y, Y\rangle / J$ is cyclic. In particular, $J$ contains $G^{\prime}$, and then since $G / G^{\prime}$ has order 1 or 3 , we must have $G / J \cong C_{3}$. Thus $|G|=3|J|=12$, giving $k=m=3$ and $G \cong A_{4}$.

Next, suppose instead that $G$ is not solvable. Since $G$ is almost Sylow-cyclic, Theorem 2.5 implies that $G$ has a subgroup $G_{0}$ of index at most 2 such that $G_{0}$ is isomorphic to $H \times L$, where $H \cong C_{u} \rtimes C_{v}$ (a semi-direct product of $C_{u}$ by $C_{v}$ ) and $L \cong \operatorname{SL}(2, q)$ 
or $\operatorname{PSL}(2, q)$, for some prime $q>3$, and that $u, v$ and $|L|$ are pairwise coprime. Clearly $H$ is a characteristic subgroup of $G_{0}$ and hence is a normal subgroup of $G$, and also its cyclic subgroup of order $u$ is normal in $G$; but on the other hand, Lemma 2.7 shows $G$ has no cyclic normal subgroup, so $H$ is trivial. Similarly, if $L \cong \operatorname{SL}(2, q)$ then $L$ contains a unique involution, which then generates a cyclic normal subgroup of $G$, again not allowed by Lemma 2.7. Thus $G_{0}=L \cong \operatorname{PSL}(2, q)$.

Finally, we use the fact that the order of any element of $\operatorname{PSL}(2, q)$ divides $q,(q-1) / 2$ or $(q+1) / 2$. Let $\varepsilon=\left|G: G_{0}\right|$, so that $|G|=\varepsilon|\operatorname{PSL}(2, q)|=\varepsilon(q-1) q(q+1) / 2$. Now $G_{0} \cong \operatorname{PSL}(2, q)$ contains the elements $X^{\varepsilon}$ (of order $k / \varepsilon$ ) and $Y$ (of odd order $m$ ), and therefore $k / \varepsilon \leq q$ and $m \leq q$. But also $|G|=2 \mathrm{~km}, 4 \mathrm{~km} / 3$ or $4 \mathrm{~km}$, so the odd prime $q$ must divide $k$ or $m$, and hence either $k=q \varepsilon$ and $m \leq(q+1) / 2$, or $m=q$ and $k \leq(q+1) \varepsilon / 2$. In both cases, $k m \leq q(q+1) \varepsilon / 2$, and therefore

$$
\varepsilon(q+1) q(q-1) / 2=|G| \leq 4 k m \leq 2 q(q+1) \varepsilon,
$$

which implies $(q-1) / 2 \leq 2$, so $q=5$. In particular, the inequalities above become equalities, so $|G|=4 \mathrm{~km}=2 q(q+1) \varepsilon$. Thus $G$ has type (D), making $k$ odd, and so $\left|G: G_{0}\right|=\varepsilon=1$, giving $\{k, m\}=\{(q+1) / 2, q\}=\{3,5\}$ and $G \cong \operatorname{PSL}(2,5) \cong A_{5}$.

We will now allow the possibility that the group $G$ is degenerate.

Theorem 4.5. Suppose G is XY-disjoint, and of type (B), (C) or (D), and let L be the normal subgroup $\operatorname{Co}(X) \operatorname{Co}(Y)$. Then $L$ is cyclic, and $G / L$ is non-degenerate, so $G / L \cong$ $S_{4}, A_{4}$ or $A_{5}$ respectively. In particular, $G$ is non-solvable only for type (D).

Proof. First, observe that $m$ is odd, so by Lemma 2.6. $\operatorname{Co}(X)$ is central in $G$, and therefore $L$ is abelian. In fact, since $\operatorname{gcd}(k, m)$ is odd and $G$ is almost Sylow-cyclic, $L$ is cyclic. Moreover, $Y$ centralizes $L$ (since $\operatorname{Co}(X)$ is central), and by Lemma 2.6 we know that $X^{2}$ centralizes $\operatorname{Co}(Y)$, and so $X^{2}$ centralizes $L$. For types (C) and (D), we know $k$ is odd, and hence also $X$ centralizes $L$ for those two types.

Now let $\bar{G}=G / L$ and let $\bar{X}$ and $\bar{Y}$ be the images of $X$ and $Y$ in $\bar{G}$. We will show that the $X$ - and $Y$-cores of $\bar{G}$ are trivial, so $\bar{G}$ is non-degenerate. Suppose $\bar{Y}^{s}$ generates the $Y$-core of $\bar{G}$. Then $K=\left\langle Y^{s}\right\rangle L$ is normal in $G$, and abelian since $Y$ centralizes $L$, and cyclic since $\operatorname{gcd}(k, m)$ is odd and $G$ is almost Sylow-cyclic. Since $K$ is cyclic, $\left\langle Y^{s}\right\rangle$ is characteristic in $K$, and therefore normal in $G$, so $\left\langle Y^{s}\right\rangle \subseteq \operatorname{Co}(Y)$. This tells us that the $Y$-core of $\bar{G}$ is trivial. Similarly, if $\bar{X}^{r}$ generates the $X$-core of $\bar{G}$, then $J=\left\langle X^{r}\right\rangle L$ is normal in $G$, and is generated by $X^{r}$ and $\operatorname{Co}(Y)=\left\langle Y^{v}\right\rangle$, say. The same argument as above shows that if $X^{r}$ centralizes $L$ then $\left\langle X^{r}\right\rangle \subseteq \operatorname{Co}(X)$ and so $\bar{X}^{r}$ is trivial. In particular, this holds for types (C) and (D), where $X$ centralizes $L$, and also for type (B) when $r$ is even. Otherwise, if $G$ has type (B) and $r$ is odd, then $|G / J|=2 r v$, where $r$ and $v$ are both odd, and $G / J$ is $(r, v, 2)$-generated; but then the Euler characteristic of the associated orientable surface is $2 r v / r-2 r v / 2+2 r v / v=2 v-r v+2 r$, which is odd, contradiction. Hence $r$ must be even when $G$ has type (B), and it follows that the $X$-core of $\bar{G}$ is trivial, for all three types. 
Thus $G / L=\bar{G}$ is non-degenerate, and so by Theorem $4.4, G / L \cong S_{4}, A_{4}$ or $A_{5}$. Finally, as $L$ is a cyclic normal subgroup of $G$, we see that $G$ is solvable if and only if $G / L \cong S_{4}$ or $A_{4}$, and hence if and only if $G$ has type (B) or (C).

Lemma 4.6. For every odd positive integer $v$, the group with presentation $\langle X, Y| X^{4}$ $\left.=Y^{3 v}=(X Y)^{2}=\left[X^{2}, Y^{3}\right]=1\right\rangle$ is an extension of $C_{v}$ by $S_{4}$, of order $24 v$, and in this group, $X Y^{3} X^{-1}=Y^{-3}$.

Proof. In the given group $G$, let $K$ be the subgroup generated by the elements $Y^{3}$ and $X Y^{3} X^{-1}$ (each of order dividing $v$ ). Note that $X$ normalizes $K$, with $X^{2}$ centralizing $K$, since $\left[X^{2}, Y^{3}\right]=1$ and then $X Y^{3} X^{-1}=X^{-1} Y^{3} X$. As $(Y X)^{2}=1$ we have $Y\left(X Y^{3} X^{-1}\right) Y^{-1}=X^{-1} Y^{-1} Y^{3} Y X=X^{-1} Y^{3} X=X Y^{3} X^{-1}$, so $Y$ centralizes $K$, and therefore $K$ is normal in $G$. Also $K$ is abelian (because $Y$ centralizes $X Y^{3} X^{-1}$ ), and so $|K|$ divides $v^{2}$. Furthermore, the quotient $G / K$ (obtained by adding the relation $Y^{3}=1$ ) is isomorphic to the ordinary $(4,3,2)$ triangle group $S_{4}$, so $K$ has index 24 in $G$. The pre-image of the Klein 4-subgroup $V_{4}$ of $S_{4}$ is an abelian normal subgroup $L$ of $G$, generated by $K$ and conjugates of $X^{2}$, and has order $4 v$. The Sylow 2-subgroup $P$ of $L$ is characteristic in $L$ and therefore normal in $G$, and since $P$ contains $X^{2}$ the quotient $G / P$ is dihedral. It follows that $X Y^{3} X^{-1}=w Y^{-3}$ for some $w \in P$, but then $w=X Y^{3} X^{-1} Y^{3}$ lies in $K \cap P$ which is trivial (since $v$ is odd), so $X Y^{3} X^{-1} Y^{3}=1$.

Lemma 4.7. The group $\left\langle X, Y \mid(X Y)^{2}=Y^{3}=\left[X^{4}, Y\right]=1\right\rangle$ has order 144, and is a central product of $C_{6}$ by $S_{4}$.

Proof. First, clearly $X^{4}$ is central, and the quotient of this group by $\left\langle X^{4}\right\rangle$ is the ordinary $(4,3,2)$ triangle group $S_{4}$. Hence all we need to do is prove that $X^{4}$ has order 6 . Let $H$ be the subgroup generated by $u=X^{2}$ and $v=\left(X Y^{-1}\right)^{2}$, which has index 6 , with Schreier transversal $\left\{1, Y, Y^{-1}, X, X Y, X Y^{-1}\right\}$. By Reidemeister-Schreier theory [13], we find that $H$ has presentation $\left\langle u, v \mid u^{2} v^{2}=u^{2}\left(u v^{-1}\right)^{2}=\left(u^{-1} v\right)^{2} v^{2}=1\right\rangle$. (In fact the 3rd relation is redundant.) In this group, $u^{2}$ is central, and $v^{-1} u v=\left(u^{-1} v\right)^{-2} u^{-1} v^{2}=$ $v^{2} u^{-1} v^{2}=u^{-5}$, so $u^{2}=v^{-1} u^{2} v=\left(v^{-1} u v\right)^{2}=\left(u^{-5}\right)^{2}=u^{-10}$, so $u^{12}=1$. Thus $X$ has order 24 , as required.

Theorem 4.8. Suppose $G$ is XY-disjoint, and of type (B), (C) or (D), and $G$ is degenerate. Then $G$ is of type (B), and has a presentation of one of these two forms:

(B1) $G \cong\left\langle X, Y \mid X^{4}=Y^{3 v}=(X Y)^{2}=\left[X^{2}, Y^{3}\right]=1\right\rangle$, or

(B2) $G \cong\left\langle X, Y \mid X^{8}=Y^{3 v}=(X Y)^{2}=\left[X^{4}, Y\right]=\left[X^{2}, Y^{3}\right]=1\right\rangle$.

In particular, $X Y^{3} X^{-1}=Y^{-3}$ in both of these two cases, so that if $G$ has type (B1) with $v>1$ or type (B2) then $G$ is degenerate. On the other hand, if $G$ is $X Y$-disjoint of type (C) or (D), or type (B1) with $v=1$, then $G$ is non-degenerate.

Proof. Again let $L=\operatorname{Co}(X) \operatorname{Co}(Y)$, which by Theorem 4.5 is cyclic.

If $G$ has type (B), then by Theorem 4.5 we know $G / L \cong S_{4}$, so that $\operatorname{Co}(X)=\left\langle X^{4}\right\rangle$ and $\operatorname{Co}(Y)=\left\langle Y^{3}\right\rangle$. Now by Lemma 2.6 $\left[X^{2}, Y^{3}\right]=1$ and $\left\langle X^{4}\right\rangle=\operatorname{Co}(X)$ is central in $G$. It follows from Lemma 4.7 that in the quotient $G / \operatorname{Co}(Y)=G /\left\langle Y^{3}\right\rangle$, the image of $\left\langle X^{4}\right\rangle$ 
has order dividing 6. $\operatorname{But} \operatorname{gcd}(k, m)=1$, so $\operatorname{gcd}(k, 3)=1$, so $\operatorname{Co}(X)$ has order 1 or 2 , and hence $k=4$ or 8 . If $k=4$, then we have the presentation in Lemma 4.6 for $G$, giving case (B1) and the relation $X Y^{3} X^{-1}=Y^{-3}$. On the other hand, if $k=8$, with $\operatorname{Co}(X)=\left\langle X^{4}\right\rangle$, then $G / \operatorname{Co}(X)$ has presentation of the form (B1), and so $X Y^{3} X^{-1}=Y^{-3}$ or $Y^{-3} X^{4}$. The latter is impossible, however, because $Y^{-3} X^{4}$ has even order (with $X^{4}$ being central), while the conjugate $X Y^{3} X^{-1}$ of $Y^{3}$ has odd order $m / 3$. Thus we obtain the presentation in case (B2), and the relation $X Y^{3} X^{-1}=Y^{-3}$.

If $G$ has type (C), then $G / L \cong A_{4}$, so $\operatorname{Co}(X)=\left\langle X^{3}\right\rangle$ and $\operatorname{Co}(Y)=\left\langle Y^{3}\right\rangle$, and $G / G^{\prime} \cong C_{3}$. Also, $L$ is central (as shown in the proof of Theorem 4.5), so by Schur's theorem, the exponent of $G^{\prime}$ divides $|G: L|=12$. Hence the orders $k / 3$ and $m / 3$ of $X^{3}$ and $Y^{3}$ divide 12, so divide 3. But $k \neq 9$, for otherwise every Sylow 3-subgroup of $G$ would contain $\operatorname{Co}(X)$ as its only subgroup of order 3 , so $\langle Y\rangle$ would intersect $\operatorname{Co}(X)$ non-trivially, contradiction. Similarly $m \neq 9$, so $\{k, m\}=\{3,3\}$, making $\operatorname{Co}(X) \operatorname{Co}(Y)$ trivial and $G$ non-degenerate.

Finally, if $G$ has type (D), then $G / L \cong A_{5}$. Again $L$ is central (as in the proof of Theorem 4.5, and so by Schur's theorem, the exponent of $G^{\prime}$ divides $|G: L|=60$. But here $G=G^{\prime}$, so $k$ and $m$ divide 60 , and as $k$ and $m$ are odd and $\operatorname{gcd}(k, m)=1$, the only possibility is $\{k, m\}=\{3,5\}$, making $G$ non-degenerate.

To summarize, we have the following possibilities when $G$ is $X Y$-disjoint. Here by 'additional relations' we mean relations which define the group $G=\operatorname{Aut}^{o}(M)$ when taken together with the standard relations $X^{k}=Y^{m}=(X Y)^{2}=1$. We also add (for later use in Section 8 ) the case (A0) of the trivial group, which is the automorphism group of a 1 -vertex, 0 -edge, 1-face map on the sphere.

Table 1. Classification of $X Y$-disjoint cases

\begin{tabular}{lccccl}
\hline Case & Type & Genus & $|G|$ & Conditions & Additional relations \\
\hline A0 & - & 0 & 1 & - & - \\
A1 & $\{2, v\}$ & 0 & $2 v$ & $v$ odd & - \\
A2 & $\{2 u, 2 v\}$ & $(u-1)(v-1)$ & $4 u v$ & $\operatorname{gcd}(u, v)=1$ & {$\left[X^{2}, Y^{2}\right]=1$} \\
B1 & $\{4,3 v\}$ & $3(v-1)$ & $24 v$ & $v$ odd & {$\left[X^{2}, Y^{3}\right]=1$} \\
B2 & $\{8,3 v\}$ & $9 v-7$ & $48 v$ & $v$ odd & {$\left[X^{4}, Y\right]=\left[X^{2}, Y^{3}\right]=1$} \\
C & $\{3,3\}$ & 0 & 12 & - & - \\
D & $\{3,5\}$ & 0 & 60 & - & - \\
\hline
\end{tabular}

Note that in each case, there is one map for every choice of the given parameters. Moreover, all these maps are reflexible, because the presentation for $G$ in each case is preserved by an involutory automorphism $\tau$ that inverts the generators $X$ and $Y$. The underlying graphs of the non-trivial maps and their duals are covers of cycles or dipoles in case (A), the octahedron or cube in case (B), the tetrahedron in case (C), and the dodecahedron or icosahedron in case (D). Simplicity (or otherwise) of the underlying graphs will be considered in the next section. 


\section{Maps with simple underlying graphs}

We can now give our main theorems about degeneracy:

Theorem 5.1. Suppose $M$ is an orientably-regular map of genus $g=p+1$, where $p$ is prime. If the underlying graph of at least one of $M$ and its dual $M^{*}$ is simple, then up to duality, one of the following holds:

(a) $p \equiv 1 \bmod 6$, and $M$ is chiral, of type $\{6,6\}$,

(b) $p \equiv 1 \bmod 8$, and $M$ is chiral, of type $\{8,8\}$,

(c) $p \equiv 1 \bmod 10$, and $M$ is chiral, of type $\{5,10\}$,

(d) $p \equiv 5 \bmod 6$, and $M$ is reflexible, of type $\{4, p+4\}$,

(e) $p=2$, and $M$ is reflexible, of type $\{3,7\},\{3,8\},\{3,12\},\{4,6\}$ or $\{4,8\}$,

(f) $p=3$, and $M$ is reflexible, of type $\{3,12\},\{4,5\},\{4,6\}$ or $\{5,5\}$,

(g) $p=5$, and $M$ is reflexible, of type $\{3,10\},\{4,6\}$ or $\{5,10\}$

(h) $p=7$, and $M$ is reflexible, of type $\{3,8\}$,

(i) $p=13$, and $M$ is reflexible, of type $\{3,7\}$.

Moreover, in cases (b) and (c) the underlying graphs of both $M$ and $M^{*}$ are simple, while in cases (a) and (d), one of them is simple while the other is not. Up to equivalence and duality the numbers of such maps are as follows: one chiral pair for each $p$ in cases (a) and (b), two chiral pairs for each $p$ in case (c), one map for each $p$ in case (d), one map of each given type in cases (e) to (g), two maps in case (h), and three maps in case (i).

Corollary 5.2. Suppose $M$ is an orientably-regular map of genus $g=p+1$, where $p$ is prime. If $M$ is chiral and $p-1$ is not divisible by 5 or 8 , or if $M$ is reflexible and $p>13$, then either $M$ or $M^{*}$ has multiple edges. Moreover, if $M$ is reflexible, $p>13$ and $p \equiv 1$ mod 6, then both $M$ and $M^{*}$ have multiple edges.

Proof (of 5.1). Let $G=\operatorname{Aut}^{o}(M)$, and suppose that $G$ is not doubly-degenerate. Then $G$ is $X Y$-disjoint. If $p$ divides $|G|$, then by Theorem 3.1, we know that either $M$ is chiral and one of cases (a) to (c) holds, or otherwise $p \leq 13$. In the latter case, we find easily that $M$ or $M^{*}$ has to be one of the reflexible maps R3.1 (of type $\{3,7\}$ ), R3.2 (of type $\{3,8\}$ ), R3.3 (of type $\{3,12\}$ ), R3.4 (of type $\{4,6\}$ ), R3.5 (of type $\{4,8\}$ ), R4.1 (of type $\{3,12\}$ ), R4.2 (of type $\{4,5\}$ ), R4.3 (of type $\{4,6\}$ ), R4.6 (of type $\{5,5\}$ ), R6.1 (of type $\{3,10\}$ ), R6.2 (of type $\{4,6\}$ ), R6.6 (of type $\{5,10\}$ ), R8.1 (of type $\{3,8\}$ ), R8.2 (of type $\{3,8\}$ ), R14.1 (of type $\{3,7\}$ ), R14.2 (of type $\{3,7\}$ ) or R14.3 (of type $\{3,7\}$ ) from the tables in [7]. On the other hand, if $|G|$ is coprime to $p=g-1$, then the theorems in Section 4 apply. For type (A) we have no examples of genus $g>1$, since $X^{2}$ and either $Y$ or $Y^{2}$ always generate a normal subgroup of $G$. For type (B), the examples of type $\{4,3 v\}$ are singly-degenerate, and reflexible, with genus $g=3 v-3$ for $v$ odd, so $p=g-1=3 v-4 \equiv 5 \bmod 6$; these give the maps in case (d) in the statement of the theorem. On the other hand, the examples of type $\{8,3 v\}$ are doubly-degenerate. Finally, for types (C) and (D) the only examples are $A_{4}$ and $A_{5}$, with genus 0 . 


\section{Reflexibility}

Next, we prove our main results about reflexibility:

Theorem 6.1. Suppose $M$ is an orientably-regular map of genus $g=p+1$, where $p$ is prime. If $M$ is chiral, then $p$ divides $|\operatorname{Aut}(M)|$ and $M$ or its dual $M^{*}$ belongs to one of the three families of maps of types $\{6,6\},\{8,8\}$ or $\{5,10\}$ from Theorem 3.1 and $p \equiv 1$ mod 6, 8 or 10 respectively. In all other cases, $M$ is reflexible.

Proof. Let $G=\operatorname{Aut}^{o}(M)$ be the group of orientation-preserving automorphisms of $M$. If $p$ divides $|G|$, then by Theorem 3.1 and inspection of the tables in [7], we know that either $M$ or $M^{*}$ belongs to one of the three families of chiral maps from Theorem 3.1, or otherwise $M$ is a reflexible map of genus at most 14. On the other hand, suppose $|G|$ is coprime to $p=g-1$. If $G$ is $X Y$-disjoint, then we may consider types (A) to (D) from Section 4 in turn; in each case, the group $G$ has a defining presentation in which every relation is of the form $X^{k}=1, Y^{m}=1,(X Y)^{2}=1,\left[X^{2}, Y^{2}\right]=1,\left[X^{2}, Y^{3}\right]=1$, or $\left[X^{4}, Y\right]=1$, but these relations are all preserved by an automorphism $\tau$ that inverts the two generators $X$ and $Y$, and so the corresponding map is always reflexible. When $G$ is not $X Y$-disjoint, its cyclic normal subgroup $N=\langle X\rangle \cap\langle Y\rangle$ is generated by some element of the form $X^{i}$ which coincides with $Y^{j}$ (for some $i$ and $j$ ), and then a presentation for $G$ can be obtained from one for $G / N$ in a way that ensures that every relation is of the form $X^{k}=1, Y^{m}=1,(X Y)^{2}=1,\left[X^{2}, Y^{2}\right]=1,\left[X^{4}, Y\right]=1,\left[X^{2}, Y^{3}\right]=1$, or $X^{i}=Y^{j}$ for some $i, j$, and again every relation of one of these forms is preserved by an automorphism $\tau$ that inverts the two generators $X$ and $Y$, and so the corresponding map is always reflexible. (Full details will be given in Section 8 .)

Corollary 6.2. There is no orientably-regular but chiral map of genus $g=p+1$, where $p$ is a prime such that $p-1$ is not divisible by 3,5 or 8 .

\section{Non-orientable regular maps of negative prime characteristic}

Let $M$ be a non-orientable regular map of type $\{k, m\}$. Then its automorphism group $G=\operatorname{Aut}(M)$ is generated by three involutions $a, b, c$ satisfying the relations $(a b)^{k}=$ $(b c)^{m}=(c a)^{2}=1$ (among others), and further, $G=\langle a, b, c\rangle=\langle X, Y\rangle$ where $X=a b$ and $Y=b c$. Conjugation by $b$ is an (inner) automorphism of $G$ that inverts each of $X$ and $Y$-and, similarly, conjugation by $a$ inverts $X$ and $X Y$, and conjugation by $c$ inverts $Y$ and $X Y$. Conversely, if $G$ is any finite group generated by elements $X$ and $Y$ of orders $k$ and $m$ for which $X Y$ has order 2 , and $G$ contains an element of order 2 that conjugates any two of $X, Y$ and $X Y$ to their respective inverses, then $G$ is the automorphism group of such a non-orientable regular map $M$.

If $M$ has Euler characteristic $-p$ for some odd prime $p$, then its orientable double cover $\tilde{M}$ of $M$ has the same type $\{k, m\}$ but its Euler characteristic is $2 \chi(M)=-2 p$, so $\tilde{M}$ has genus $p+1$, and its full automorphism group is $G \times C_{2}$, with $G$ preserving orientation. Hence our observations from earlier sections apply to $G$ acting on $\tilde{M}$. In 
particular, since $\tilde{M}$ is not chiral, we find that either $p \leq 13$, or otherwise $p$ does not divide $|G|$.

The non-orientable regular maps of genus 2 to 15 are given in [7], and from there we find that the only examples of small negative prime characteristic are N4.1 (of type $\{4,6\}$ ), N4.2 (of type $\{4,6\}$ ), N5.1 (of type $\{4,5\}$ ), N5.2 (of type $\{4,6\}$ ), N5.3 (of type $\{5,5\}$ ), N5.4 (of type $\{6,6\}$ ), N7.1 (of type $\{4,6\}$ ), N7.2 (of type $\{4,9\}$ ), N9.1 (of type $\{3,8\}$ ), N9.2 (of type $\{3,8\}$ ), N9.3 (of type $\{6,10\}$ ), N13.1 (of type $\{4,15\}$ ), N13.2 (of type $\{6,14\}$ ), and N15.1 (of type $\{3,7\}$ ).

Lemma 7.1. For $p$ odd, the group $G$ has no central involution, so $G$ is $X Y$-disjoint.

Proof. It is easy to check that this holds for the above examples of small characteristic. Hence we may suppose that $p$ does not divide $|G|$, and then $G$ is almost Sylow-cyclic. Now the subgroup $\langle a, c\rangle$ (which stabilizes an edge of $M$ ) is isomorphic to $C_{2} \times C_{2}$, and so by almost Sylow-cyclicity of $G$, every involution in $G$ must be conjugate to one of $a, c$ or $a c$. The involutions $a$ and $c$ are not central in $G$, for otherwise $X=a b$ or $Y=b c$ would have order 2, giving $k=2$ or $m=2$, which is impossible when $M$ has characteristic $-p$. Also $a c=X Y$ is not central, for otherwise $G=\langle X Y, Y\rangle$ would be abelian. Hence $G$ has no central involution. Finally, consider $N=\langle X\rangle \cap\langle Y\rangle$. This is a central cyclic subgroup of $G$, but also each of its elements is inverted under conjugation by $b$, so it has order 1 or 2. Since $G$ has no central involutions, we conclude that $N$ is trivial, and thus $G$ is $X Y$-disjoint.

It follows that all examples other than those of small genus can be found from the families of maps that we considered in Section 4

For type (A), we need $\operatorname{gcd}(k, m)=2$, for otherwise $k=2$ and so the map is planar (and therefore orientable). Thus $k=2 r$ and $m=2 s$ with $\operatorname{gcd}(r, s)=1$, and $G$ is an extension of a cyclic group of order $r s$ by $C_{2} \times C_{2}$, with presentation $\langle X, Y| X^{2 r}=Y^{2 s}=$ $\left.(X Y)^{2}=\left[X^{2}, Y^{2}\right]=1\right\rangle$. Now in this group, each of $X^{2}$ and $Y^{2}$ generates a cyclic normal subgroup, which by the above lemma contains no involution, so $r$ and $s$ are both odd. The Euler-Poincaré formula now implies that $p=-\chi(M)=|G| / 4-|G| / 2 k-|G| / 2 m=$ $r s-s-r=(r-1)(s-1)-1$ is congruent to $3 \bmod 4$. On the other hand, since $r$ is odd and $X^{2} Y=Y X^{-2}$ (by Lemma 4.2), we have $X^{r} Y X^{r}=X Y X^{-(r-1)} X^{r}=X Y X=Y^{-1}$, so conjugation by the involution $X^{r}$ inverts $Y$. Similarly, conjugation by the involution $Y^{s}$ inverts $X$. It follows that $X^{r} Y^{s}$ is an involution that conjugates each of $X$ and $Y$ to its inverse, and hence one such non-orientable map of type $\{2 r, 2 s\}$ and characteristic $r+s-r s$ exists for all odd positive integers $r$ and $s$. The maps $\mathrm{N} 9.3$ (of type $\{6,10\}$ ) and N13.2 (of type $\{6,14\}$ ) are examples.

For type (B), we need $k=4$ (since $X^{k / 2}$ cannot be central), and so $G$ is an extension of a cyclic group of odd order $v$ by $\operatorname{PSL}(2,3) \cong S_{4}$, with presentation $\langle X, Y$ | $\left.X^{4}=Y^{3 v}=(X Y)^{2}=\left[X^{2}, Y^{3}\right]=1\right\rangle$. The Euler-Poincaré formula implies that $p=-\chi(M)=|G| / 4-|G| / 8-|G| / 6 v=3 v-4$ is congruent to $5 \bmod 6$. On the other hand, consider $T=Y X^{2} Y^{-1}$, which (as a conjugate of $X^{2}$ ) is an involution. Now also $Y^{-2} X$ is an involution, since the relations $Y^{3} X=X Y^{-3}$ (from Lemma 4.6) and $(Y X)^{2}=1$ imply $Y^{-2} X=Y Y^{-3} X=Y X Y^{3}=X^{-1} Y^{2}=\left(Y^{-2} X\right)^{-1}$. Thus 
$T X=Y X^{2} Y^{-1} X=X^{-1} Y^{-1} X Y^{-1} X$, being conjugate to $Y^{-2} X$, is an involution, so $T X T=X^{-1}$. Similarly $T X Y$ is an involution as well, since $T X Y=X^{-1} T Y=$ $X^{-1} Y X^{2}=X^{-1}(Y X) X$, and therefore $T X Y T=(X Y)^{-1}$. Hence $T$ is an involution that inverts each of $X$ and $X Y$ by conjugation, and it follows that one such non-orientable map of type $\{4,3 v\}$ and characteristic $4-3 v$ exists for every odd integer $v$. In fact these maps are members of the family constructed in Example 3.1 of [8], with $Y X^{2} Y^{-1}$ giving the 'inner reflector' in the quotient $S_{4}$, and they include the maps N7.2 (of type $\{4,9\}$ ) and N13.1 (of type $\{4,15\}$ ).

Finally, once again for types (C) and (D) the only examples have $G \cong A_{4}$ and $A_{5}$, which are impossible for negative Euler characteristic.

Thus we have the following, most of which was proved in [5]:

Theorem 7.2. Suppose $M$ is a non-orientable regular map of characteristic $-p$, where $p$ is prime. Then up to duality, one of the following holds:

(a) $p \equiv 3 \bmod 4$, and $M$ has type $\{2 r, 2 s\}$, for $r$, s odd and $p=r s-s-r$,

(b) $p \equiv 5 \bmod 6$, and $M$ has type $\{4, p+4\}$,

(c) $p=2$, and $M$ has type $\{4,6\}$,

(d) $p=3$, and $M$ has type $\{4,5\},\{4,6\},\{5,5\}$ or $\{6,6\}$,

(e) $p=5$, and $M$ has type $\{4,6\}$,

(f) $p=7$, and $M$ has type $\{3,8\}$,

(g) $p=13$, and $M$ has type $\{3,7\}$.

Moreover, there is just one such map in each of cases (a), (b), (d), (e) and (g), while there are two in cases (c) and (f). Hence in particular, if $p$ is prime, $p>13$ and $p \equiv 1 \bmod 12$, then there exists no non-orientable regular map of characteristic $-p$.

\section{The full classification}

In this section, we complete the classification of orientably-regular maps $M$ for which $\left|\operatorname{Aut}^{O}(M)\right|$ is coprime to $g-1$, where $g$ is the genus of $M$, and hence obtain a full classification of all orientably-regular maps of genus $p+1$ where $p$ is prime.

As previously, we suppose $M$ has type $\{k, m\}$, and let $X$ and $Y$ be generators of $G=\operatorname{Aut}^{o}(M)$ satisfying $X^{k}=Y^{m}=(X Y)^{2}=1$, and also we let $N=\langle X\rangle \cap\langle Y\rangle$. The condition that $|G|$ is coprime to $g-1$ implies that $g$ is even, and that $G$ is almost Sylow-cyclic. Moreover, the quotient $G / N$ is $X Y$-disjoint, and so from Section 4 we have a classification of all the possibilities for $G / N$ when this is non-trivial, with specific presentations for $G / N$ in each case.

Here we drop the assumption that $G$ is $X Y$-disjoint, to allow the possibility that $N$ is non-trivial. Let $n=|N|$ and $h=|G / N|$, and then let $k=r n$ and $m=s n$, so that $r$ and $s$ are the orders of the images of $X$ and $Y$ in the quotient $G / N$. When $G / N$ is of type (A2), we may suppose without loss of generality that $v=s / 2$ is odd, and so can take $v$ to be odd in all four of the first cases from Table 1 in Section 4

With the above notation assumed, we will use the following: 
Lemma 8.1. If $h>1$ then $X^{h} Y^{h}=1$.

Proof. Consider the transfer homomorphism $f: G \rightarrow N$, given by $x \mapsto x^{|G: N|}=x^{h}$ for all $x \in G$. If $h>1$ then $h=|G / N|$ is even (by what we found in Section 4), and as $X Y$ has order 2 it follows that $1=(X Y)^{h}=f(X Y)=f(X) f(Y)=X^{h} Y^{h}$.

Lemma 8.2. Let $C$ be a cyclic group of order $a b$, where $a, b>1$, and let $B$ be its unique subgroup of order $b$ and index $a$. Suppose $C$ has an (involutory) automorphism that fixes $B$ and inverts each element of the quotient $C / B$. Then $\operatorname{gcd}(a, b)=1$ or 2 . Hence, in particular, if $a$ or $b$ is odd then $\operatorname{gcd}(a, b)=1$. If, however, $b$ is even and $a$ is divisible by 4 , then $b / 2$ is odd and $\operatorname{gcd}(a, b / 2)=1$.

Proof. The given automorphism $\alpha$ of $C$ takes $x \mapsto x^{e}$ (for all $x \in C$ ) for some $e$ coprime to $|C|=a b$. Since $\alpha$ fixes $B$ we have $a e \equiv a \bmod a b$, and since $\alpha$ inverts each element of $C / B$ we have $e \equiv j a-1 \bmod a b$ for some $j$. From these two congruences we find that $(j a-2) a \equiv(e-1) a \equiv 0 \bmod a b$, and hence $j a-2$ is a multiple of $b$. This implies that $\operatorname{gcd}(a, b)$ divides 2 , and the rest follows easily.

Corollary 8.3. If $G / N$ is of type (A1), (A2), (B1) or (B2), as in Table 1 (with $v$ odd in case (A2)), then $\operatorname{gcd}(v, n)=1$; moreover, if $G / N$ is of type (A2), then $\operatorname{gcd}(u, n)=1$ whenever $u$ or $n$ is odd.

Proof. In each case, $v$ is odd, and $\langle Y\rangle$ contains a normal cyclic subgroup of order $v n$, containing $N$. Call this subgroup $C$. Then since $X=(X Y) Y^{-1}$, conjugation by $X$ is an involutory automorphism of $C$, that induces the inversion mapping on $C / N$ (since $G / N$ is dihedral in case (A1), and $X Y^{2} X^{-1}=Y^{-2} \bmod N$ in case (A2), and $X Y^{3} X^{-1}=Y^{-3}$ $\bmod N$ in cases (B1) and (B2)). As $N$ is central, Lemma 8.2 applies, with $B=N$ and $(a, b)=(v, n)$, and since $v$ is odd, this gives $\operatorname{gcd}(v, n)=1$. Similarly, if $u$ or $n$ is odd in case (A2), then $\operatorname{gcd}(u, n)=1$.

We can now proceed to the full classification:

Theorem 8.4. Suppose $M$ is an orientably-regular map of type $\{k, m\}$ and genus $g$ with orientation-preserving automorphism group $G=\operatorname{Aut}^{o}(M)$, having the property that $|G|$ is coprime to $g-1$. Then up to duality, one of the following holds:

(A0) $k=m=n,|G|=n$, and $g=n / 4$, for some $n \equiv 0 \bmod 8$,

(A1) $k=2 n, m=v n,|G|=2 v n$, and $g=v(n-1) / 2$, for some $v$ and $n$ such that $v$ is odd, $n \equiv 1 \bmod 4$, and $\operatorname{gcd}(v, n)=\operatorname{gcd}(v+2, n)=1$,

(A2) $k=2 u n, m=2 v n,|G|=4 u v n$, and $g=u v n-(u+v)+1$, for some $u, v$ and $n$ such that $\operatorname{gcd}(u, v)=\operatorname{gcd}(u+v, n)=1$, and either $n$ is odd and $\operatorname{gcd}(u v, n)=1$, or $n \equiv 2 \bmod 4$ and $\operatorname{gcd}(u v, n / 2)=1$,

(B1) $k=4 n, m=3 v n,|G|=24 v n$, and $g=6 v n-3 v-3$ for some $v$ and $n$ such that $v$ is odd, and $\operatorname{gcd}(3 v, n)=\operatorname{gcd}(3 v+4, n)=1$, and $n \neq 0 \bmod 4$,

(B2) $k=8 n, m=3 v n,|G|=48 v n$, and $g=12 v n-3 v-7$ for some $v$ and $n$ such that $v$ and $n$ are both odd, and $\operatorname{gcd}(3 v, n)=\operatorname{gcd}(3 v+8, n)=1$,

(C) $k=m=3 n,|G|=12 n$, and $g=3 n-3$ for some odd $n$,

(D) $k=3 n, m=5 n,|G|=60 n$, and $g=15 n-15$ for some $n$ coprime to 30 . 
Moreover, there is just one such map for every choice of the parameters in each case, and all of these maps are reflexible. Presentations for $G$ are given in Table 2.

Table 2. Presentations for $G=\operatorname{Aut}^{o}(M)$ when $|G|$ is coprime to $g-1$

\begin{tabular}{|c|c|c|}
\hline & Type & Additional relations in defining presentation for $G$ \\
\hline (A0) & $\{n, n\}$ & $X Y=Y^{n / 2}$ \\
\hline (A1) & $\{2 n, v n\}$ & $X^{2 v} Y^{2 v}=1$ \\
\hline (A2) & $\{2 u n, 2 v n\}$ & {$\left[X^{2}, Y^{2}\right]=X^{4 u v} Y^{4 u v}=1$, if $n$ is odd, } \\
\hline (B1) & $\{4 n, 3 v n\}$ & $\begin{array}{l}\text { or }\left[X^{2}, Y^{2}\right]=X^{24 v} Y^{24 v}=1, \text { if } n \text { is odd, } \\
{\left[X^{2}, Y^{3}\right]=X^{24 v} Y^{24 v}, Y^{24 v}=1, X^{2 n}=Y^{3 v n / 2}, \text { if } n \equiv 2 \bmod 4} \\
\text { or }\left[X^{2}, Y^{3}\right]=X^{24 v} Y^{24}=1 \text { if } 4\end{array}$ \\
\hline (B2) & $\{8 n, 3 v n\}$ & {$\left[X^{4}, Y\right]=\left[X^{2}, Y^{3}\right]=X^{48 v} Y^{48 v}=1$} \\
\hline (C) & $\{3 n, 3 n\}$ & $X^{12} Y^{12}=1$ \\
\hline (D) & $\{3 n, 5 n\}$ & $X^{60} Y^{60}=1$ \\
\hline
\end{tabular}

Proof. First, suppose $|G / N|=h=1$. Then $G=N$ is cyclic, and $k=m=n=|G|$, and the Euler-Poincaré formula gives $g-1=(m / 2-1-1) / 2=m / 4-1$, so $m / 4$ is even, say $m=8 t$. Hence the map has type $\{8 t, 8 t\}$ and genus $2 t$. Also $X Y=Y^{4 t}$ and hence $X=Y^{4 t-1}$, so clearly there is one such map for each positive integer $t$. This accounts for item (A0).

From now on, we suppose $h>1$. In all cases, the genus $g$ can be calculated from the Euler-Poincare formula, and many of the conditions on the parameters follow immediately from that (and the assumption that $|G|$ is coprime to $g$ ). Also a relation of the form $X^{h} Y^{h}=1$ in each case follows from Lemma 8.1, and the relations $\left[X^{2}, Y^{2}\right]=1$ (in case (A2)) and $\left[X^{2}, Y^{3}\right]=1$ (in cases (B1) and (B2)) follow from Lemma 2.6 .

In case (A1) from Section 4, we have $h=2 v$ and $(r, s)=(2, v)$, where $v$ is odd. In this case $2(g-1)=v(n-1)-2$, and as this must be twice an odd integer, we find $n \equiv 1 \bmod 4$. Also Corollary 8.3 tells us that $\operatorname{gcd}(v, n)=1$. The relation $X^{2 v} Y^{2 v}=1$ (from Lemma 8.1 can be rewritten as $\left(X^{2}\right)^{v}=\left(Y^{v}\right)^{2}$, and because $\operatorname{gcd}(n, 2 v)=1$, this expresses $X^{2}$ as a power of $Y^{v}$ in $N$ and vice versa.

In case (A2), we have $h=4 u v$ and $(r, s)=(2 u, 2 v)$, with $\operatorname{gcd}(2 u, v)=1$.

If $n$ is odd, then Corollary $8.3 \operatorname{gives} \operatorname{gcd}(u, n)=\operatorname{gcd}(v, n)=1, \operatorname{so} \operatorname{gcd}(u v, n)=1$. Accordingly, the relation $X^{4 u v} Y^{4 u v}=1$ rewritten as $\left(X^{2 u}\right)^{2 v}\left(Y^{2 v}\right)^{2 u}=1$ expresses $X^{2 u}$ as a power of $Y^{2 v}$ in $N$, and vice versa. On the other hand, if $n$ is even, then $\operatorname{gcd}(v, n)=1$ by Corollary 8.3 , and also $u$ is even, since $\operatorname{gcd}(u+v, n)=1$ by the genus calculation. Next, as the 2-part of $|G|$ divides $2 u n$, some power of $X$ generates a cyclic group $C$ of index 2 in a Sylow 2-subgroup $H$ of $G$, and conjugation by an element $z$ of $H \backslash C$ is an involutory automorphism $\alpha$ of $C$ that centralizes $B=C \cap N$. Moreover, in the dihedral quotient $G /\left\langle Y^{2}\right\rangle$, the image of $z$ inverts all elements of the image of $C$, which is $C\left\langle Y^{2}\right\rangle /\left\langle Y^{2}\right\rangle \cong C /\left(C \cap\left\langle Y^{2}\right\rangle\right)=C / B$. Thus Lemma 8.2 applies, with $a=|C / B|$ divisible by 4 since $u$ is even, and with $b$ as the 2-part of $n$. It follows that $b / 2$ is odd, so $n / 2$ is odd, hence $\operatorname{gcd}(2 u, n / 2)=1$, and thus $\operatorname{gcd}(h, n / 2)=\operatorname{gcd}(2 u v, n / 2)=1$. When 
taken together with the obvious relation $X^{u n}=Y^{v n}$ (giving the central involution of $N$ ), the relation $X^{4 u v} Y^{4 u v}=1$ rewritten as $\left(X^{2 u}\right)^{2 v}\left(Y^{2 v}\right)^{2 u}=1$ is now enough to express $X^{2 u}$ and $Y^{2 v}$ in terms of each other in $N$. This gives the presentations for type (A2) in Table 2 .

In case (B1), we have $h=24 v$ and $(r, s)=(4,3 v)$, where $v$ is odd. The relation $X^{24 v} Y^{24 v}=1$ tells us that $\left(X^{4}\right)^{6 v}$ and $\left(Y^{3 v}\right)^{8}$ are mutually inverse elements of $N$. As these have orders $n / \operatorname{gcd}(n, 6 v)$ and $n / \operatorname{gcd}(n, 8)$ respectively, we find that $\operatorname{gcd}(n, 6 v)=$ $\operatorname{gcd}(n, 8)$, which must be at most 2. In particular, this implies that $\operatorname{gcd}(n, 3 v)=1$. If $n$ is odd, then $X^{24 v} Y^{24 v}=1$ expresses $X^{4}$ as a power of $Y^{3 v}$ and vice versa. On the other hand, if $n$ is even, then as in case (A2) some power of $X$ generates a cyclic group $C$ having index 2 in a Sylow subgroup $H$ of $G$, and conjugation by an element of $H \backslash C$ is an involutory automorphism $\alpha$ of $C$ that centralizes $B=C \cap N$. Here $\alpha$ inverts all elements of $C / B$ since a Sylow 2-subgroup of the quotient $G / N \cong S_{4}$ is dihedral. By Lemma 8.2 with $a=|C / B|=4$, we find that $n / 2$ is odd. When taken together with the obvious relation $X^{2 n}=Y^{3 v n / 2}$ (giving the central involution of $N$ ), the relation $\left(X^{4}\right)^{6 v}\left(Y^{3 v}\right)^{8}=1$ is now enough to express $X^{4}$ and $Y^{3 v}$ in terms of each other in $N$.

In case (B2), we have $h=48 v$, with $r=8$ and $s=3 v$, where $v$ is odd. The relation $1=X^{48 v} Y^{48 v}=\left(X^{8}\right)^{6 v}\left(Y^{3 v}\right)^{16}$ tells us that $\operatorname{gcd}(n, 6 v)=\operatorname{gcd}(n, 12)$, which must be at most 2, and hence $\operatorname{gcd}(n, 3 v)=1$. Here $\operatorname{Co}(X)=\left\langle X^{4}\right\rangle$ and $\operatorname{Co}(Y)=\left\langle Y^{3}\right\rangle$, and Lemma 2.6 shows that $Y^{2}$ centralizes $\operatorname{Co}(X)$ and $X^{2}$ centralizes $\operatorname{Co}(Y)$. It follows that $X^{4}$ commutes with both $Y^{2}$ and $Y^{3}$, and so commutes with $Y$. Thus $X^{4}$ is central. Now we can use Lemma 8.2 applied to a Sylow 2-subgroup $C$ of $\langle X\rangle$ and a subgroup $B$ of index 4 in $C$ (containing $C \cap\left\langle X^{4}\right\rangle$ ), with $b$ as the 2-part of $2 n$, and $\operatorname{get} \operatorname{gcd}(4, b / 2)=1$, from which it follows that $n$ is odd. In particular, $Y$ has odd order, so $X^{48 v} Y^{48 v}=1$ expresses $X^{8}$ as a power of $Y^{3 v}$ and vice versa.

In case (C), we have $h=12$ and $r=s=3$. The genus calculation shows $n$ is odd, and the relation $X^{12} Y^{12}=1$ tells us that $X^{3}$ and $Y^{3}$ are elements of $N$ whose 4th powers are mutually inverse (and in fact implies $X^{3} Y^{3}=1$ ). This gives item $(\mathrm{g})$.

Similarly, in case (D), where $h=12$ and $(r, s)=(3,5)$, we find that $n$ is odd (by the genus calculation). The relation $X^{60} Y^{60}=1$ gives $\left(X^{3}\right)^{20}=\left(Y^{5}\right)^{-12}$ (and in fact implies $\left.X^{15} Y^{15}=1\right)$. This expresses $X^{3}$ and $Y^{5}$ in terms of each other in $N$, and also gives $\operatorname{gcd}(n, 5)=1$ and $\operatorname{gcd}(n, 3)=1$, so $\operatorname{gcd}(n, 30)=1$. In particular, by SchurZassenhaus, $G \cong C_{n} \times A_{5}$.

This completes the classification. Reflexibility of the maps follows from the fact that the relations in the defining presentations for the group $G=\operatorname{Aut}^{o}(M)$ in each case are all preserved by replacing $X$ and $Y$ by their inverses.

Finally, we have the following consequence of this classification:

Corollary 8.5. Suppose $M$ is an orientably-regular map of genus $p+1$ where $p$ is prime. Then up to duality:

(a) $M$ is one of the chiral maps described in Theorem 3.1 or

(b) $M$ is one of the reflexible maps described in Theorem 8.4 or

(c) $M$ is one of the reflexible maps of genus 3, 4, 6, 8, 12 or 14 listed in [7] and not already included in case (b). 
Acknowledgments. Research of M. D. E. Conder was supported in part by the N.Z. Marsden Fund, Grants UOA 316 and UOA 412.

Research of J. Širáň was supported in part by the N.Z. Marsden Fund, Grant UOA 316 and the Slovak APVV Grant 104-07.

\section{References}

[1] Accola, R. D.: Topics in the Theory of Riemann Surfaces. Lecture Notes in Math. 1595, Springer (1994) Zbl 0820.30002 MR 1329541

[2] Belolipetsky, M., Jones, G. A.: Automorphism groups of Riemann surfaces of genus $p+1$, where $p$ is prime. Glasgow Math. J. 47, 379-393 (2005) Zbl 1084.30050 MR 2203507

[3] Bosma, W., Cannon, J., Playoust, C.: The Magma Algebra System I: The User Language. J. Symbolic Comput. 24, 235-265 (1997) Zbl 0898.68039 MR 1484478

[4] Brahana, H. R.: Regular maps and their groups. Amer. J. Math. 49, 268-284 (1927) JFM 53.0549.01 MR 1506619

[5] Breda d'Azevedo, A., Nedela, R., Širáň, J.: Classification of regular maps of negative prime Euler characteristic. Trans. Amer. Math. Soc. 357, 4175-4190 (2005) Zbl 1065.05033 MR 2159705

[6] Conder, M. D. E.: Regular maps and hypermaps of Euler characteristic -1 to -200. J. Combin. Theory Ser. B 99, 455-459 (2009) Zbl pre05523281 MR 2482963

[7] Conder, M., Dobcsányi, P.: Determination of all regular maps of small genus. J. Combin. Theory Ser. B 81, 224-242 (2001) (with associated lists of computational data available at http://www.math.auckland.ac.nz/ conder/hypermaps.html) Zbl 1028.05045 1 MR 1814906

[8] Conder, M., Everitt, B.: Regular maps on non-orientable surfaces. Geom. Dedicata 56, 209219 (1995) Zbl 0832.05053 MR 1338960

[9] Conder, M., Potočnik, P., Širáň, J.: Regular maps with almost Sylow-cyclic automorphism groups, and classification of regular maps with Euler characteristic $-p^{2}$. Preprint

[10] Coxeter, H. S. M., Moser, W. O. J.: Generators and Relations for Discrete Groups. 4th ed., Springer, Berlin (1980) Zbl 0422.20001 MR 0562913

[11] Grothendieck, A.: Esquisse d'un programme. Preprint, Montpellier (1984); also in: London Math. Soc. Lecture Note Ser. 242, Cambridge Univ. Press, 5-48 (1997) Zbl 0901.14001

[12] Ito, N.: Über das Produkt von zwei abelschen Gruppen. Math Z. 62, 400-401 (1955) Zbl 0064.25203 MR 0071426

[13] Johnson, D. L.: Presentations of Groups. 2nd ed., Cambridge Univ. Press (1997) Zbl 0906.20019 MR 1472735

[14] Jones, G. A.: Maps on surfaces and Galois groups. Math. Slovaca 47, 1-33 (1997) Zbl 0958.05036 MR 1476746

[15] Jones, G. A., Singerman, D.: Belyı̆ functions, hypermaps, and Galois groups. Bull. London Math. Soc. 28, 561-590 (1996) Zbl 0853.14017 MR 1405488

[16] Robinson, D. J. S.: A Course in the Theory of Groups. 2nd ed., Springer (1996) Zbl 0836.20001 MR 1357169

[17] Suzuki, M.: On finite groups with cyclic Sylow subgroups for all odd primes. Amer. J. Math. 77, 657-691 (1955) Zbl 0068.25601 MR 0074411

[18] Wilson, S. E., Breda d'Azevedo, A.: Surfaces having no regular hypermaps. Discrete Math. 277, 241-274 (2004) Zbl 1033.05029 MR 2033735

[19] Wong, W. J.: On finite groups with semi-dihedral Sylow 2-subgroups. J. Algebra 4, 52-63 (1966) MR 0210779 